\title{
Siphons in Chemical Reaction Networks
}

\author{
Anne Shiu*, Bernd Sturmfels \\ Dept. of Mathematics, University of California, Berkeley, CA 94720, USA
}

Received: 29 April 2009 / Accepted: 7 January 2010 / Published online: 21 January 2010

(C) The Author(s) 2010. This article is published with open access at Springerlink.com

\begin{abstract}
Siphons in a chemical reaction system are subsets of the species that have the potential of being absent in a steady state. We present a characterization of minimal siphons in terms of primary decomposition of binomial ideals, we explore the underlying geometry, and we demonstrate the effective computation of siphons using computer algebra software. This leads to a new method for determining whether given initial concentrations allow for various boundary steady states.
\end{abstract}

Keywords Chemical reaction systems $\cdot$ Siphon $\cdot$ Steady state $\cdot$ Monomial ideal Binomial ideal · Primary decomposition

\section{Introduction}

In systems biology, a population model or chemical reaction system is said to be "persistent" if none of its species can become extinct if all species are present at the initial time. Those subsets of the species that can be absent in steady state are called "siphons." Angeli et al. (2007) suggested the concept of siphons to study the long-term behavior of dynamical systems that model chemical reactions. In terms of the dynamics, a siphon is the index set of a forward-invariant face of the positive orthant. Any boundary steady state must lie in the interior of such a face. Hence, to investigate the trajectories, it is useful to list all minimal siphons. The present paper offers an algebraic characterization of siphons, and it shows how this translates into a practical tool for computing siphons.

Following Adleman et al. (2008) and Craciun et al. (2009), we represent a chemical reaction network as a directed graph $G$ whose nodes are labeled by monomials and whose edges correspond to reactions. A siphon of $G$ is a nonempty subset $Z$ of the variables such that, for every directed edge $m \rightarrow m^{\prime}$ in $G$, whenever one of the variables in the monomial $m^{\prime}$ lies in $Z$ then so does at least one of the variables in $m$. In Section 2 we relate this definition to the description of siphons given in Angeli et al. (2007) and Cordone et al. (2005), we review the underlying dynamics, and we discuss its meaning in terms of polyhedral geometry. Our algebraic approach is presented in

\footnotetext{
${ }^{*}$ Corresponding author.

E-mail addresses: annejls@math.berkeley.edu (Anne Shiu),bernd@math.berkeley.edu (Bernd Sturmfels).
} 
Section 3. Theorem 3.1 expresses the minimal siphons of $G$ in terms of the primary decomposition of a binomial ideal associated to $G$. If the directed graph $G$ is strongly connected then the ideal encoding the minimal siphons is generated by squarefree monomials. In Theorem 3.2 and Algorithm 3.6, we explain how to compute the relevant (stoichiometrically compatible) siphons for any set of initial conditions. In particular, a chemical reaction system without relevant siphons has no boundary steady states, and this property is sufficient for proving persistence in many systems (Angeli et al., 2007; Siegel and MacLean, 2004). In Section 4, we demonstrate that the relevant computations can be performed effectively using computer algebra software, such as Macaulay 2 (Grayson and Stillman).

In the remainder of the Introduction, we present three examples from the systems biology literature, with the aim of illustrating our algebraic representation of chemical reaction networks and the computation of siphons.

Example 1.1. We consider a receptor-ligand dimer model, which is analyzed by Chavez in her thesis (Chavez, 2003, §7.2) and by Anderson (2008, Example 4.1):

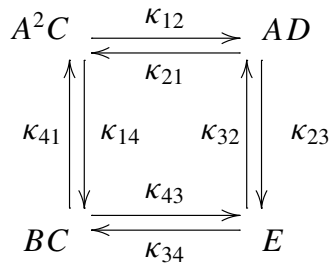

Note that the reaction $A^{2} C \leftrightarrows A D$ is usually denoted by $2 A+C \leftrightarrows A+D$. The biochemical species are as follows: the species $A$ denotes a receptor, $B$ denotes a "dimer" state of $A$ (two receptors joined together), and $C$ denotes a ligand that can bind either to $A$ (to form $D$ ) or to $B$ (to form $E$ ). There are three minimal siphons, $\{A, B, E\},\{A, C, E\}$, and $\{C, D, E\}$, which correspond to the minimal primes of the monomial ideal of the complexes $\left\langle A^{2} C, A D, E, B C\right\rangle$. We will return to this example in Section 4.

Example 1.2. The following enzymatic mechanism was analyzed by Siegel and MacLean (2004), and also by Chavez (2003, Example 4.6.1):

$$
\begin{aligned}
& S E \leftrightarrows Q \leftrightarrows P E \leftrightarrows \\
& Q I \leftrightarrows R .
\end{aligned}
$$

The species are $S$ (a substrate), $E$ (an enzyme), $P$ (a product), $I$ (an uncompetitive inhibitor), and intermediate complexes $Q$ and $R$. Here, the graph consists of two strong components, and we encode it in the binomial ideal $\langle S E-Q, Q-P E, Q I-R\rangle+$ $\langle E P Q R S\rangle$. The radical of this ideal equals

$$
\langle E, Q, R\rangle \cap\langle I, R, E S-Q, P-S\rangle \cap\langle P, Q, R, S\rangle .
$$

By Theorem 3.1, the minimal siphons are the variables in these prime ideals. Thus, the minimal siphons are $\{E, Q, R\},\{I, R\}$, and $\{P, Q, R, S\}$. 
Example 1.3. Here is the network for a basic one-step conversion reaction:

$$
\begin{gathered}
S_{0} E \leftrightarrows X \rightarrow P E \\
P F \leftrightarrows Y \rightarrow S_{0} F
\end{gathered}
$$

The enzyme $E$ helps convert a substrate $S_{0}$ into a product $P$, and a second enzyme $F$ reverts the product $P$ back into the original enzyme $S_{0}$; these are also called "futile cycles" (Angeli and Sontag, 2006; Manrai and Gunawardena, 2008). Such reactions include phosphorylation and de-phosphorylation events, and they take place in MAPK cascades. This network has three minimal siphons: $\{E, X\},\{F, Y\}$, and $\left\{P, S_{0}, X, Y\right\}$. To see this algebraically, we form the binomial ideal

$$
\left\langle E S_{0}-X, X(E P-X), F P-Y, Y\left(F S_{0}-Y\right), E F P S_{0} X Y\right\rangle .
$$

This ideal corresponds to $\mathfrak{T}_{G}$ in Theorem 3.1, and it has six minimal primes:

$$
\begin{aligned}
& \left\langle E, X, F S_{0}-Y, P-S_{0}\right\rangle, \quad\left\langle F, Y, P-S_{0}, S_{0} E-X\right\rangle, \quad\left\langle P, S_{0}, X, Y\right\rangle, \\
& \langle E, X, Y, F\rangle, \quad\langle E, X, P, Y\rangle, \quad \text { and } \quad\left\langle F, S_{0}, X, Y\right\rangle .
\end{aligned}
$$

The three minimal siphons arise from the first three of these six primes.

\section{Reaction networks, siphons, and steady states}

A chemical reaction network is defined by a finite labeled directed graph $G$ with $n$ vertices. The $i$ th vertex of $G$ is labeled with a monomial $c^{y_{i}}=c_{1}^{y_{i 1}} c_{2}^{y_{i 2}} \cdots c_{s}^{y_{i s}}$ in $s$ unknowns $c_{1}, \ldots, c_{s}$, and an edge $(i, j)$ is labeled by a positive parameter $\kappa_{i j}$. This graph defines the ordinary differential equations

$$
\frac{d c}{d t}=\Psi(c) \cdot A_{\kappa} \cdot Y
$$

where $\Psi(c)=\left(c^{y_{1}}, c^{y_{2}}, \ldots, c^{y_{n}}\right)$ is the row vector of the monomials, $Y=\left(y_{i j}\right)$ is the $n \times s$-matrix of exponent vectors of the $n$ monomials, and $A_{\kappa}$ is the $n \times n$-matrix whose off-diagonal entries are the $\kappa_{i j}$ and whose row sums are zero (i.e., minus the Laplacian of $G$ ). The equations (1) are those of mass-action kinetics, although the concept of a siphon is independent of the choice of kinetics. In order for each chemical complex $c^{y_{i}}$ to be a reactant or product of at least one reaction, we assume that $G$ has no isolated points. For a complex $c^{y_{i}}$ and for $a \in[s]$, we write $c_{a} \mid c^{y_{i}}$ (" $c_{a}$ divides $c^{y_{i}}$ ") if $y_{i a}>0$; in other words the $i$ th complex contains species $a$. If the $i$ th complex does not contain species $a$, then we write $c_{a} \nmid c^{y_{i}}$.

A nonempty subset $Z$ of the index set $[s]:=\{1,2, \ldots, s\}$ is a siphon if for all $z \in Z$ and all reactions $c^{y_{i}} \longrightarrow c^{y_{j}}$ with $c_{z} \mid c^{y_{j}}$, there exists $a \in Z$ such that $c_{a} \mid c^{y_{i}}$. Siphons were called "semilocking sets" in Anderson (2008) and Anderson and Shiu (2010). Note that the set of siphons of $G$ does not depend on the choice of parameters $\kappa_{i j}$.

With any nonempty subset $Z \subset[s]$ we associate the prime ideal

$$
\mathfrak{P}_{Z}:=\left\langle c_{a}: a \in Z\right\rangle
$$


in the polynomial ring $\mathbb{Q}\left[c_{1}, c_{2}, \ldots, c_{s}\right]$. Recall (e.g., from Cox et al., 2007) that the $v a$ riety of $\mathfrak{P}_{Z}$, denoted by $V\left(\mathfrak{P}_{Z}\right)$, is the set of points $x \in \mathbb{R}^{s}$ such that $f(x)=0$ for all polynomials $f \in \mathfrak{P}_{Z}$. Thus, the nonnegative variety $V_{\geq 0}\left(\mathfrak{P}_{Z}\right)$ is the face of the positive orthant $\mathbb{R}_{\geq 0}^{s}$ defined by all $Z$-coordinates being zero.

Proposition 2.1. A nonempty subset $Z$ of $[s]$ is a siphon if and only if $V_{\geq 0}\left(\mathfrak{P}_{Z}\right)$ is forward-invariant with respect to the dynamical system (1).

Proof: This is the content of Proposition 2 in Angeli et al. (2007).

In Example 1.1, the dynamical system (1) takes the explicit form

$$
\begin{aligned}
& d A / d t=-\left(\kappa_{12}+2 \kappa_{14}\right) A^{2} C+\left(\kappa_{21}-\kappa_{23}\right) A D+\kappa_{32} E+2 \kappa_{41} B C, \\
& d B / d t=\kappa_{14} A^{2} C-\left(\kappa_{41}+\kappa_{43}\right) B C+\kappa_{34} E \\
& d C / d t=-\kappa_{12} A^{2} C+\kappa_{21} A D-\kappa_{43} B C+\kappa_{34} E \\
& d D / d t=\kappa_{12} A^{2} C-\left(\kappa_{21}+\kappa_{23}\right) A D+\kappa_{32} E \\
& d E / d t=\kappa_{23} A D+\kappa_{43} B C-\left(\kappa_{32}+\kappa_{34}\right) E .
\end{aligned}
$$

This is a dynamical system on $\mathbb{R}_{\geq 0}^{5}$. Each of the three minimal siphons $\{A, B, E\}$, $\{A, C, E\}$, and $\{C, D, E\}$ defines a two-dimensional face of $\mathbb{R}_{\geq 0}^{5}$. For example, $V_{\geq 0}\left(\mathfrak{P}_{\{A, B, E\}}\right)$ is the face in which the coordinates $A, B$, and $E$ are zero and $C$ and $D$ are nonnegative. The minimality of the three siphons implies that no face of dimension three or four is forward-invariant.

We next collect some results relating siphons to boundary steady states, that is, nonnegative steady states of (1) having at least one zero-coordinate. These connections are behind our interest in computing siphons. See Anderson (2008), Anderson and Shiu (2010), Angeli et al. (2007) for details on how siphons relate to questions of persistence (the property that positive trajectories of (1) have no accumulation points on the boundary of the orthant $\mathbb{R}_{\geq 0}^{s}$ ). We first show that a boundary steady state necessarily lies in the relative interior of a face $V_{\geq 0}\left(\mathfrak{P}_{Z}\right)$ indexed by a siphon $Z$.

Lemma 2.2. Fix a reaction network $G$, and let $\gamma$ be a point on the boundary of the positive orthant $\mathbb{R}_{\geq 0}^{s}$ with zero coordinate set $Z:=\left\{i \in[s]: \gamma_{i}=0\right\}$. If $\gamma$ is a boundary steady state of (1), then the index set $Z$ is a siphon.

Proof: Assume that $c_{z} \mid c^{y_{j}}$ for some species $z \in Z$ and some complex $c^{y_{j}}$ of $G$. Let $\mathcal{I}$ index complexes that react to $c^{y_{j}}$ but do not contain the species $z$ :

$$
\mathcal{I}:=\left\{i \in[n]: c^{y_{i}} \longrightarrow c^{y_{j}} \text { is a reaction of } G \text { and } c_{z} X c^{y_{i}}\right\} .
$$

Then we have

$$
\left.\frac{d c_{z}}{d t}\right|_{c=\gamma}=\sum_{i \in \mathcal{I}} \kappa_{i j} y_{j z} \gamma^{y_{i}}=0,
$$


where the second equality holds because $\gamma$ is a steady state. The summands of (2) are nonnegative, so we have $\gamma^{y_{i}}=0$ for all $i \in \mathcal{I}$. Thus, if $i \in \mathcal{I}$ there exists $a_{i} \in[s]$ with $\gamma_{a_{i}}=0$ (so, $a_{i} \in Z$ ), and hence $c_{a_{i}} \mid c^{y_{i}}$.

A similar result holds for boundary $\omega$-limit points (accumulation points) of a trajectory; see Anderson (2008), Angeli et al. (2007) or Anderson and Shiu (2010, Theorem 2.14). We are interested in the dynamics arising from some initial condition $c^{(0)} \in \mathbb{R}_{>0}^{s}$, so we restrict our attention to polyhedra, called invariant polyhedra, of the following form:

$$
\mathcal{P}_{c^{(0)}}:=\left(c^{(0)}+L_{\text {stoi }}\right) \cap \mathbb{R}_{\geq 0}^{s} .
$$

Here, $L_{\text {stoi }}:=\operatorname{span}\left\{y_{j}-y_{i}: c^{y_{i}} \rightarrow c^{y_{j}}\right.$ is a reaction $\}$ is the stoichiometric subspace in $\mathbb{R}^{s}$. For any $c$ and any $\kappa$, the right-hand side vector $\Psi(c) \cdot A_{\kappa} \cdot Y$ of the dynamical system (1) lies in $L_{\text {stoi }}$ and, therefore, the polyhedron $\mathcal{P}_{c^{(0)}}$ is forward-invariant with respect to (1). For any index set $W \subset[s]$, let

$$
F_{W}:=\left\{x \in \mathcal{P}_{c^{(0)}}: x_{i}=0 \text { if } i \in W\right\}=V_{\geq 0}\left(\mathfrak{P}_{W}\right) \cap \mathcal{P}_{c^{(0)}}
$$

denote the corresponding (possibly empty) face of $\mathcal{P}_{c^{(0)}}$. All faces of $\mathcal{P}_{c^{(0)}}$ have this form; see Anderson and Shiu $(2010, \$ 2.3)$ for further details. Lemma 2.2 implies the following: Given an invariant polyhedron $\mathcal{P}_{c^{(0)}}$, if all siphons $Z$ yield empty faces, $F_{Z}=\emptyset$, then $\mathcal{P}_{c^{(0)}}$ contains no boundary steady states. In Theorem 3.5, we shall present an algebraic method for deciding when this happens.

We now examine the case when the chemical reaction network is strongly connected, i.e., between any two complexes there is a sequence of reactions.

Lemma 2.3. Assume that $G$ is strongly connected. Then a point $\gamma \in \mathbb{R}_{\geq 0}^{s}$ is a boundary steady state if and only if $Z=\left\{i \in[s]: \gamma_{i}=0\right\}$ is a siphon.

Proof: The forward implication is Lemma 2.2. Now let $\gamma$ be a boundary point whose zero-coordinate set $Z$ is a siphon. Because $G$ is strongly connected, all complexes $c^{y_{i}}$ evaluated at $\gamma$ are zero $\left(\gamma^{y_{i}}=0\right)$, and hence each monomial that appears on the righthand side of (1) vanishes at $c=\gamma$.

From a polyhedral geometry point of view, Lemma 2.3 states the following: For strongly connected reaction networks $G$, any face of an invariant polyhedron $\mathcal{P}_{c}(0)$ either has no steady states in its interior or the entire face consists of steady states. We shall see now that a similar result holds for toric dynamical systems. Recall (from Craciun et al., 2009) that (1) is a toric dynamical system if the parameters $\kappa_{i j}$ are such that $\Psi(c) \cdot A_{\kappa}=0$ has a positive solution $c \in \mathbb{R}_{>0}^{s}$ (which is called a complex-balancing steady state). The following result concerns the faces of invariant polyhedra of toric dynamical systems.

Lemma 2.4. Let $c^{(0)} \in \mathbb{R}_{>0}^{s}$ be a positive initial condition of a toric dynamical system. Then a face $F_{Z}$ of the invariant polyhedron $\mathcal{P}_{c^{(0)}}$ contains a steady state in its interior if and only if $Z$ is a siphon.

Proof: This is derived in Anderson (2008, Lemma 2.8) albeit in different language. 


\section{Binomial ideals and monomial ideals}

In what follows, we characterize the minimal siphons of a chemical reaction network $G$ in the language of combinatorial commutative algebra (Miller and Sturmfels, 2005). It will be shown that they arise as components in primary decompositions. For any initial conditions $c^{(0)}$, we characterize those siphons that define nonempty faces of the invariant polyhedron $\mathcal{P}_{c^{(0)}}$. In the next section, we shall see that these results translate into a practical new method for enumerating siphons.

Throughout this section, we fix the ring $R=\mathbb{Q}\left[c_{1}, \ldots, c_{s}\right] /\left\langle c_{1} c_{2} \ldots c_{s}\right\rangle$. This is the ring of polynomial functions with $\mathbb{Q}$-coefficients on the union of the coordinate hyperplanes in $\mathbb{R}^{s}$. All our ideals will live in this ring.

With a given network $G$, we associate the following three ideals in $R$ :

$$
\begin{aligned}
\mathfrak{T}_{G} & =\left\langle c^{y_{i}} \cdot\left(c^{y_{j}}-c^{y_{i}}\right): c^{y_{i}} \rightarrow c^{y_{j}} \text { is a reaction of } G\right\rangle, \\
\mathfrak{J}_{G} & =\left\langle c^{y_{j}}-c^{y_{i}}: c^{y_{i}} \rightarrow c^{y_{j}} \text { is a reaction of } G\right\rangle, \\
\mathfrak{M}_{G} & =\langle\Psi(c)\rangle=\left\langle c^{y_{1}}, c^{y_{2}}, \ldots, c^{y_{n}}\right\rangle .
\end{aligned}
$$

Thus, $\mathfrak{T}_{G}$ encodes the directed edges, and $\mathfrak{J}_{G}$ encodes the underlying undirected graph. These are pure difference binomial ideals (Dickenstein et al., 2010; Eisenbud and Sturmfels, 1996), while $\mathfrak{M}_{G}$ is the monomial ideal of the complexes. The following is our main result.

Theorem 3.1. The minimal siphons of a chemical reaction network $G$ are the inclusionminimal sets $\left\{i \in[s]: c_{i} \in \mathfrak{P}\right\}$ where $\mathfrak{P}$ runs over the minimal primes of $\mathfrak{T}_{G}$. If each connected component of $G$ is strongly connected then $\mathfrak{T}_{G}$ can be replaced in this formula by the ideal $\mathfrak{J}_{G}$. Moreover, if $G$ is strongly connected then $\mathfrak{T}_{G}$ can be replaced by the monomial ideal $\mathfrak{M}_{G}$.

Proof: The complex variety $V_{\mathbb{C}}\left(\mathfrak{T}_{G}\right)$ consists of all points $\gamma \in \mathbb{C}^{s}$ having at least one zero coordinate and satisfying $\gamma^{y_{i}} \cdot\left(\gamma^{y_{j}}-\gamma^{y_{i}}\right)=0$ for all reactions. We first claim that our assertion is equivalent to the statement that the minimal siphons are the inclusion-minimal sets of the form $\left\{i \in[s]: \gamma_{i}=0\right\}$ where $\gamma$ runs over $V_{\mathbb{C}}\left(\mathfrak{T}_{G}\right)$. Indeed if $\mathfrak{P}$ is a minimal associated prime of $\mathfrak{T}_{G}$, let $\gamma \in\{0,1\}^{s}$ be defined by $\gamma_{i}=1$ if and only if $c_{i} \notin \mathfrak{P}$. It follows that $\left\{i \in[s]: c_{i} \in \mathfrak{P}\right\}=\left\{i \in[s]: \gamma_{i}=0\right\}$ and $\gamma \in V_{\mathbb{C}}(\mathfrak{P}) \subset V_{\mathbb{C}}\left(\mathfrak{T}_{G}\right)$. Conversely, if $\gamma \in V_{\mathbb{C}}\left(\mathfrak{T}_{G}\right)$, then $\gamma \in V_{\mathbb{C}}(\mathfrak{P})$ for some minimal associated prime $\mathfrak{P}$, and so we have the containment $\left\{i \in[s]: c_{i} \in \mathfrak{P}\right\} \subset\left\{i \in[s]: \gamma_{i}=0\right\}$. If, furthermore, the set $\left\{i \in[s]: \gamma_{i}=0\right\}$ is minimal among those defined by $\gamma^{\prime} \in V_{\mathbb{C}}\left(\mathfrak{T}_{G}\right)$, then by above it must follow that the containment is in fact equality.

Next, if $\gamma$ is in $V_{\mathbb{C}}\left(\mathfrak{T}_{G}\right)$, then we can replace $\gamma$ by the $0-1$ vector $\delta$ defined by $\delta_{i}=0$ if $\gamma_{i}=0$ and $\delta_{i}=1$ if $\gamma_{i} \neq 0$. This nonnegative real vector has the same support as $\gamma$ and lies in the variety of $\mathfrak{T}_{G}$. Hence, our claim is that the minimal siphons are the inclusionminimal sets of the form $\left\{i \in[s]: \delta_{i}=0\right\}$ where $\delta$ runs over $V_{\{0,1\}}\left(\mathfrak{T}_{G}\right)$. But this is obvious because $\delta^{y_{i}} \cdot\left(\delta^{y_{j}}-\delta^{y_{i}}\right)=0$ if and only if $\delta^{y_{j}}=0$ implies $\delta^{y_{i}}=0$.

Now, the minimal associated primes of $\mathfrak{T}_{G}$ depend only on the radical of $\mathfrak{T}_{G}$, so we can replace $\mathfrak{T}_{G}$ by any other ideal that has the same radical. If the components of $G$ are strongly connected, then the complex $c^{y_{i}}$ can produce $c^{y_{j}}$ if and only if $c^{y_{j}}$ can produce 
$c^{y_{i}}$, and in this case both $c^{y_{i}} \cdot\left(c^{y_{j}}-c^{y_{i}}\right)$ and $c^{y_{j}} \cdot\left(c^{y_{i}}-c^{y_{j}}\right)$ are in $\mathfrak{T}_{G}$. Hence, the radical of $\mathfrak{T}_{G}$ contains the binomial $c^{y_{i}}-c^{y_{j}}$, and we conclude that $\mathfrak{T}_{G}$ and $\mathfrak{J}_{G}$ have the same radical.

Finally, $\mathfrak{M}_{G}$ is a monomial ideal, and associated primes of a monomial ideal are of the form $\mathfrak{P}_{Z}$ for some $Z \subset[s]$. It is straightforward to see that if $G$ is strongly connected, $\mathfrak{P}_{Z}$ contains $\mathfrak{M}_{G}$ if and only if $Z$ is a siphon.

When analyzing a concrete chemical reaction network $G$, one often is given an initial vector $c^{(0)} \in \mathbb{R}_{>0}^{s}$ for the dynamical system (1), or at least a subset $\Omega$ of $\mathbb{R}_{>0}^{s}$ that contains $c^{(0)}$. A siphon $Z \subset[s]$ of $G$ is called $c^{(0)}$-relevant if the face $F_{Z}$ of the invariant polyhedron $\mathcal{P}_{c^{(0)}}$ is nonempty. In other words, if $Z$ is $c^{(0)}$-relevant, then there exists a boundary point that is stoichiometrically compatible with $c^{(0)}$ and has zero-coordinate set containing $Z$. For any subset $\Omega$ of $\mathbb{R}_{>0}^{s}$, we say that $Z$ is $\Omega$-relevant if it is $c^{(0)}$-relevant for at least one point $c^{(0)}$ in $\Omega$. Finally, we call a siphon relevant if it is $\mathbb{R}_{>0}^{s}$-relevant. Relevant siphons are also called "critical" siphons (Angeli et al., 2007), and nonrelevant siphons are also called "stoichiometrically infeasible" siphons (Anderson and Shiu, 2010) and "structurally nonemptiable" siphons (Angeli et al., 2007). We next explain how to enlarge the ideals $\mathfrak{T}_{G}, \mathfrak{J}_{G}$, and $\mathfrak{M}_{G}$ so that their minimal primes encode only the siphons that are relevant.

We recall that the stoichiometric subspace $L_{\text {stoi }}$ of $\mathbb{R}^{s}$ is spanned by all vectors $y_{j}-y_{i}$ where $c^{y_{i}} \rightarrow c^{y_{j}}$ is a reaction in $G$. Its orthogonal complement $L_{\text {cons }}:=\left(L_{\text {stoi }}\right)^{\perp}$ is the space of conservation relations. Let $\mathcal{Q}$ denote the image of the nonnegative orthant $\mathbb{R}_{\geq 0}^{s}$ in the quotient space $\mathbb{R}^{s} / L_{\text {stoi }} \simeq L_{\text {cons. }}$. Thus $\mathcal{Q}$ is a convex polyhedral cone and its interior points are in bijection with the invariant polyhedra $\mathcal{P}_{c^{(0)}}$. Further, $\mathcal{Q}$ is isomorphic to the cone spanned by the columns of any matrix $\mathbf{A}$ whose rows form a basis for $L_{\text {cons. }}$. This isomorphism is given by the map

$$
\phi_{\mathbf{A}}: \mathcal{Q} \rightarrow\left\{\sum_{i=1}^{s} \alpha_{i} a_{i}: \alpha_{1}, \alpha_{2}, \ldots, \alpha_{s} \geq 0\right\}, \quad \bar{q} \mapsto \sum_{i=1}^{s} q_{i} a_{i},
$$

where $q=\left(q_{1}, q_{2}, \ldots, q_{s}\right) \in \mathbb{R}_{>0}^{s}$ and $a_{1}, a_{2}, \ldots, a_{s}$ are the columns of the matrix $\mathbf{A}$. For simplicity, we identify the cone $\mathcal{Q}$ with the image of $\phi_{\mathbf{A}}$. A subset $F$ of $[s]=\{1,2, \ldots, s\}$ is called a facet of $\mathcal{Q}$ if the corresponding columns of $\mathbf{A}$ are precisely the rays lying on a maximal proper face of $\mathcal{Q}$. Any maximal proper face of $\mathcal{Q}$ also is called a facet. The list of all facets of $\mathcal{Q}$ can be computed using polyhedral software such as polymake (Gawrilow and Joswig, 2000).

We represent the facets of $\mathcal{Q}$ by the following squarefree monomial ideal:

$$
\mathfrak{B}=\bigcap_{F \text { facet of } \mathcal{Q}}\left\langle c_{i}: i \notin F\right\rangle=\bigcap_{F \text { facet of } \mathcal{Q}} \mathfrak{P}_{F^{c}} .
$$

Each vertex of an invariant polyhedron $\mathcal{P}_{c^{(0)}}$ is encoded uniquely by its support $V$, which is a subset of $[s]$. Consider the squarefree monomial ideal

$$
\mathfrak{B}_{c^{(0)}}=\left\langle\prod_{i \in V} c_{i}: V \text { encodes a vertex of } \mathcal{P}_{c^{(0)}}\right\rangle .
$$


The distinct combinatorial types of the polyhedra $\mathcal{P}_{c^{(0)}}$ determine a natural chamber decomposition of the cone $\mathcal{Q}$ into finitely many smaller cones: if two polyhedra $\mathcal{P}_{c^{(0)}}$ and $\mathcal{P}_{d^{(0)}}$ correspond to points in such a chamber of the decomposition, then the polyhedra have the same set of supports $V$ of their vertices. For an example, see Fig. 1. In the context of chemical reaction networks, such as chamber decomposition appeared in recent work of Craciun et al. (2009). Specifically, its chambers were denoted $S_{i}$ in Craciun et al. (2009, Section 2.1).

The ideal $\mathfrak{B}_{c^{(0)}}$ depends only on the chamber that contains the image of $c^{(0)}$. For any subset $\Omega \subset \mathbb{R}_{>0}^{s}$, we take the sum of the ideals corresponding to all chambers that intersect the image of $\Omega$ in $\mathcal{Q}$. That sum is the ideal

$$
\mathfrak{B}_{\Omega}=\left\langle\prod_{i \in V} c_{i}: V \text { encodes a vertex of } \mathcal{P}_{c^{(0)}} \text { for some } c^{(0)} \in \Omega\right\rangle .
$$

The above ideals are considered either in the polynomial ring $\mathbb{Q}\left[c_{1}, \ldots, c_{s}\right]$ or in its quotient $R=\mathbb{Q}\left[c_{1}, \ldots, c_{s}\right] /\left\langle c_{1} c_{2} \cdots c_{s}\right\rangle$, depending on the context.

Let $\mathfrak{T}_{1}$ and $\mathfrak{T}_{2}$ be two arbitrary ideals in $R$. Recall (e.g., from Cox et al., 2007) that the saturation of $\mathfrak{T}_{1}$ with respect to $\mathfrak{T}_{2}$ is a new ideal that contains $\mathfrak{T}_{1}$, namely,

$$
\operatorname{Sat}\left(\mathfrak{T}_{1}, \mathfrak{T}_{2}\right)=\left(\mathfrak{T}_{1}: \mathfrak{T}_{2}^{\infty}\right)=\left\{f \in R: f \cdot\left(\mathfrak{T}_{2}\right)^{m} \subseteq \mathfrak{T}_{1} \text { for some } m \in \mathbb{Z}_{>0}\right\}
$$

Here, we shall be interested in the following nine saturation ideals:

$$
\begin{array}{lll}
\operatorname{Sat}\left(\mathfrak{T}_{G}, \mathfrak{B}\right), & \operatorname{Sat}\left(\mathfrak{T}_{G}, \mathfrak{B}_{c^{(0)}}\right), & \operatorname{Sat}\left(\mathfrak{T}_{G}, \mathfrak{B}_{\Omega}\right), \\
\operatorname{Sat}\left(\mathfrak{J}_{G}, \mathfrak{B}\right), & \operatorname{Sat}\left(\mathfrak{J}_{G}, \mathfrak{B}_{c^{(0)}}\right), & \operatorname{Sat}\left(\mathfrak{J}_{G}, \mathfrak{B}_{\Omega}\right), \\
\operatorname{Sat}\left(\mathfrak{M}_{G}, \mathfrak{B}\right), & \operatorname{Sat}\left(\mathfrak{M}_{G}, \mathfrak{B}_{c^{(0)}}\right), & \operatorname{Sat}\left(\mathfrak{M}_{G}, \mathfrak{B}_{\Omega}\right) .
\end{array}
$$

The following theorem is a refinement of our result in Theorem 3.1.

Theorem 3.2. The relevant minimal siphons of $G$ are the inclusion-minimal sets $\left\{i \in[s]: c_{i} \in \mathfrak{P}\right\}$ where $\mathfrak{P}$ runs over minimal primes from (4). The ideals in the first, second, and third columns yield relevant siphons, $c^{(0)}$-relevant siphons, and $\Omega$-relevant siphons, respectively. The ideals in the first row are for all networks $G$, those in the third row for strongly connected $G$, and those in the middle row for $G$ with strongly connected components.

Proof: The variety of the ideal $\operatorname{Sat}\left(\mathfrak{T}_{1}, \mathfrak{T}_{2}\right)$ is the union of all irreducible components of the variety $V\left(\mathfrak{T}_{1}\right)$ that do not lie in $V\left(\mathfrak{T}_{2}\right)$. The result now follows from Theorem 3.1 and the following observations. The nonnegative variety $V_{\geq 0}(\mathfrak{B})$ consists of all points in $\mathbb{R}_{>0}^{s}$ whose image modulo $L_{\text {stoi }}$ lies in the boundary of the cone $\mathcal{Q}$. Thus, for a minimal siphon $Z$, the image of the variety $V_{\geq 0}\left(\mathfrak{P}_{Z}\right)$ is in the boundary of $\mathcal{Q}$ if and only if $Z$ is not relevant. More precisely, the image of $V_{\geq 0}\left(\mathfrak{P}_{Z}\right)$ is in the interior of the subcone spanned by $\left\{a_{i}: i \notin Z\right\}$, so there exists a facet of $\mathcal{Q}$ that contains the subcone if and only if $Z$ is not relevant. Therefore, any irreducible component of $V\left(\mathfrak{J}_{G}\right)$ (or $V\left(\mathfrak{T}_{G}\right)$ or $V\left(\mathfrak{M}_{G}\right)$ ) defines a nonrelevant siphon $Z$ if and only if it lies in $V\left(\mathfrak{P}_{F^{c}}\right)$ for some facet $F$ of $\mathcal{Q}$, which is equivalent to lying in $V(\mathfrak{B})$. 
Next, the variety $V_{\geq 0}\left(\mathfrak{B}_{c^{(0)}}\right)$ is the union of all faces of the orthant $\mathbb{R}_{\geq 0}^{s}$ that are disjoint from the invariant polyhedron $\mathcal{P}_{c^{(0)}}$. So, for a minimal siphon $Z$, the ideal $\mathfrak{P}_{Z}$ does not contain $\mathfrak{B}_{c^{(0)}}$ if and only if there exists a vertex of $\mathcal{P}_{c^{(0)}}$ whose zero-coordinate set contains $Z$, which is equivalent to the condition that the face $F_{Z}$ of the polyhedron is nonempty. Hence, any component of $V\left(\mathfrak{J}_{G}\right)$ (or $V\left(\mathfrak{T}_{G}\right)$ or $V\left(\mathfrak{M}_{G}\right)$ ) that defines a minimal siphon $Z$ lies in $V\left(\mathfrak{B}_{c^{(0)}}\right)$ if and only if $Z$ is not relevant. Finally, the variety $V_{\geq 0}\left(\mathfrak{B}_{\Omega}\right)$ is the intersection of the varieties $V_{\geq 0}\left(\mathfrak{B}_{c^{(0)}}\right)$ as $c^{(0)}$ runs over $\Omega$.

Example 3.3. In Examples 1.2 and $1.3, \mathcal{Q}$ is the cone over a triangle, and the three minimal siphons are precisely the facets of that triangular cone. Thus, there are no relevant siphons at all. This is seen algebraically by verifying the identities $\operatorname{Sat}\left(\mathfrak{J}_{G}, \mathfrak{B}\right)=\langle 1\rangle$ and $\operatorname{Sat}\left(\mathfrak{T}_{G}, \mathfrak{B}\right)=\langle 1\rangle$.

We now discuss the case when a network has no relevant siphons, by making the connection to work of Angeli et al. (2007), which focuses on chemical reaction networks whose siphons $Z$ all satisfy the following condition:

( $\star$ ) there exists a nonnegative conservation relation $l \in L_{\text {cons }} \cap \mathbb{R}_{\geq 0}^{s}$ whose support $\operatorname{supp}(l)=\left\{i \in[s]: l_{i}>0\right\}$ is a subset of $Z$.

Recall that Angeli et al. call siphons satisfying this property "structurally nonemptiable" (Angeli et al. 2007, Section 8). Note that the property ( $\star$ ) needs only to be checked for minimal siphons in order for all siphons to satisfy the property. For some chemical reaction systems, such as toric dynamical systems (including Examples 1.1 and 1.2), this property is sufficient for proving persistence (Anderson, 2008; Anderson and Shiu, 2010; Angeli et al., 2007; Siegel and MacLean, 2004), and what was offered in this section are elegant and efficient algebraic tools for deriving such proofs.

Lemma 3.4. For a chemical reaction network $G$, a siphon $Z$ satisfies property ( $\star$ ) if and only if $Z$ is not relevant (which is equivalent to $\mathfrak{B} \subseteq \mathfrak{P}_{Z}$ ).

Proof: The "only if" direction is clear. For the "if" direction, let $Z$ be a non-relevant siphon. As usual, for $\sigma:=\operatorname{dim} L_{\text {stoic }}$, we fix a matrix $\mathbf{A} \in \mathbb{R}^{(s-\sigma) \times s}$ whose rows span $L_{\text {cons }}$, and we identify $\mathcal{Q}$ with the cone spanned by the columns $a_{i}$ of $\mathbf{A}$. Let $F$ be a facet of $\mathcal{Q}$ that contains the image of $V_{\geq 0}\left(\mathfrak{P}_{Z}\right)$, and let $v \in \mathbb{R}^{s-\sigma}$ be a vector such that the linear functional $\langle v,-\rangle$ is zero on $F$ and is positive on points of $\mathcal{Q}$ outside of $F$. The vector $l:=v \mathbf{A}$ is in $L_{\text {cons }}$, and we claim that this is a nonnegative vector as in ( $\star$ ). Indeed, $l_{i}=\left\langle v, a_{i}\right\rangle$ is zero if $i \in F$ and is positive if $i \notin F$, and thus $\operatorname{supp}(l)=F^{c} \subseteq Z$.

The following result extends Theorem 2 in Angeli et al. (2007).

Theorem 3.5. None of the siphons of the network $G$ are relevant if and only if $\operatorname{Sat}\left(\mathfrak{T}_{G}, \mathfrak{B}\right)=\langle 1\rangle$ if and only if all siphons satisfy property ( $\star$ ). In this case, none of the invariant polyhedra $\mathcal{P}_{c^{(0)}}$ has a boundary steady state.

Proof: The first claim follows from Lemma 3.4 above. The second claim follows from the definition of relevant siphons and Lemma 2.2. 
We next present a characterization of the ideals $\mathfrak{B}$ and $\mathfrak{B}_{c^{(0)}}$ in terms of combinatorial commutative algebra. This allows us to compute these ideals entirely within a computer algebra system (such as Macaulay 2, Grayson and Stillman), without having to make any calls to polyhedral software (such as polymake). We assume a subroutine that computes the largest monomial ideal contained in a given binomial ideal in the polynomial ring $\mathbb{R}\left[c_{1}, \ldots, c_{s}\right]$. Let $\mathfrak{T}_{\text {stoi }}$ and $\mathfrak{T}_{\text {cons }}$ denote the lattice ideals associated with the subspaces $L_{\text {stoi }}$ and $L_{\text {cons. }}$. These ideals are generated by the binomials $c^{u_{+}}-c^{u-}$ where $u=u_{+}-u_{-}$runs over all vectors in $\mathbb{Z}^{s}$ that lie in the respective subspace. Here, $u_{+} \in \mathbb{Z}_{\geq 0}^{s}$ and $u_{-} \in \mathbb{Z}_{\geq 0}^{s}$ denote the positive and negative parts of a vector $u$ in $\mathbb{Z}^{s}$.

Algorithm 3.6. The ideals $\mathfrak{B}$ and $\mathfrak{B}_{c^{(0)}}$ can be computed as follows:

1. The squarefree monomial ideal $\mathfrak{B}$ is the radical of the largest monomial ideal contained in $\mathfrak{T}_{\text {stoi }}+\left\langle c_{1} c_{2} \cdots c_{s}\right\rangle$.

2. The squarefree monomial ideal $\mathfrak{B}_{c^{(0)}}$ is Alexander dual to the radical of the largest monomial ideal contained in the initial ideal in $_{c(0)}\left(\mathfrak{T}_{\text {cons }}\right)$.

3. If $c^{(0)}$ is generic (i.e., the polyhedron $\mathcal{P}_{c^{(0)}}$ is simple) then the radical of $\operatorname{in}_{c^{(0)}}\left(\mathfrak{T}_{\text {cons }}\right)$ is a monomial ideal, and its Alexander dual equals $\mathfrak{B}_{c^{(0)}}$.

The correctness of part 1 rests on the fact that the zero set of the lattice ideal $\mathfrak{T}_{\text {stoi }}$ is precisely the affine toric variety associated with the cone $\mathcal{Q}$. Adding the principal ideal $\left\langle c_{1} c_{2} \cdots c_{s}\right\rangle$ to $\mathfrak{T}_{\text {stoi }}$ is equivalent to taking the image of $\mathfrak{T}_{\text {stoi }}$ in $R$. The nonnegative variety of the resulting ideal is the union of all faces of $\mathbb{R}_{\geq 0}^{s}$ whose image modulo $L_{\text {stoi }}$ is in the boundary of $\mathcal{Q}$.

For parts 2 and 3, we are using concepts and results from the textbook (Miller and Sturmfels, 2005). The key idea is to use the initial concentration vector $c^{(0)}$ as a partial term order. Initial ideals of lattice ideals are discussed in Miller and Sturmfels (2005, Section 7.4). Alexander duality of squarefree monomial ideals is introduced in Miller and Sturmfels (2005, Section 5.1). The correctness of part 3 is an immediate corollary to Miller and Sturmfels (2005, Theorem 7.33) and part 2 is derived from part 3 by a perturbation argument. In the next section, we demonstrate how to compute all these ideals in Macaulay 2.

\section{Computing siphons in practice}

We start with a network that has both relevant and nonrelevant siphons. This example serves to illustrate the various results in the previous section.

Example 4.1. We return to the chemical reaction network in Example 1.1. The sums $C+$ $D+E$ and $A+2 B+D+2 E$ are both constant along trajectories. Chemically, this says that both the total amount of free and bound forms of the ligand and the total amount of the free and bound forms of the receptor remain constant. Thus, the matrix $\mathbf{A}$ can be taken to be

$$
\mathbf{A}=\left(a_{A}, a_{B}, a_{C}, a_{D}, a_{E}\right)=\left(\begin{array}{lllll}
0 & 0 & 1 & 1 & 1 \\
1 & 2 & 0 & 1 & 2
\end{array}\right)
$$




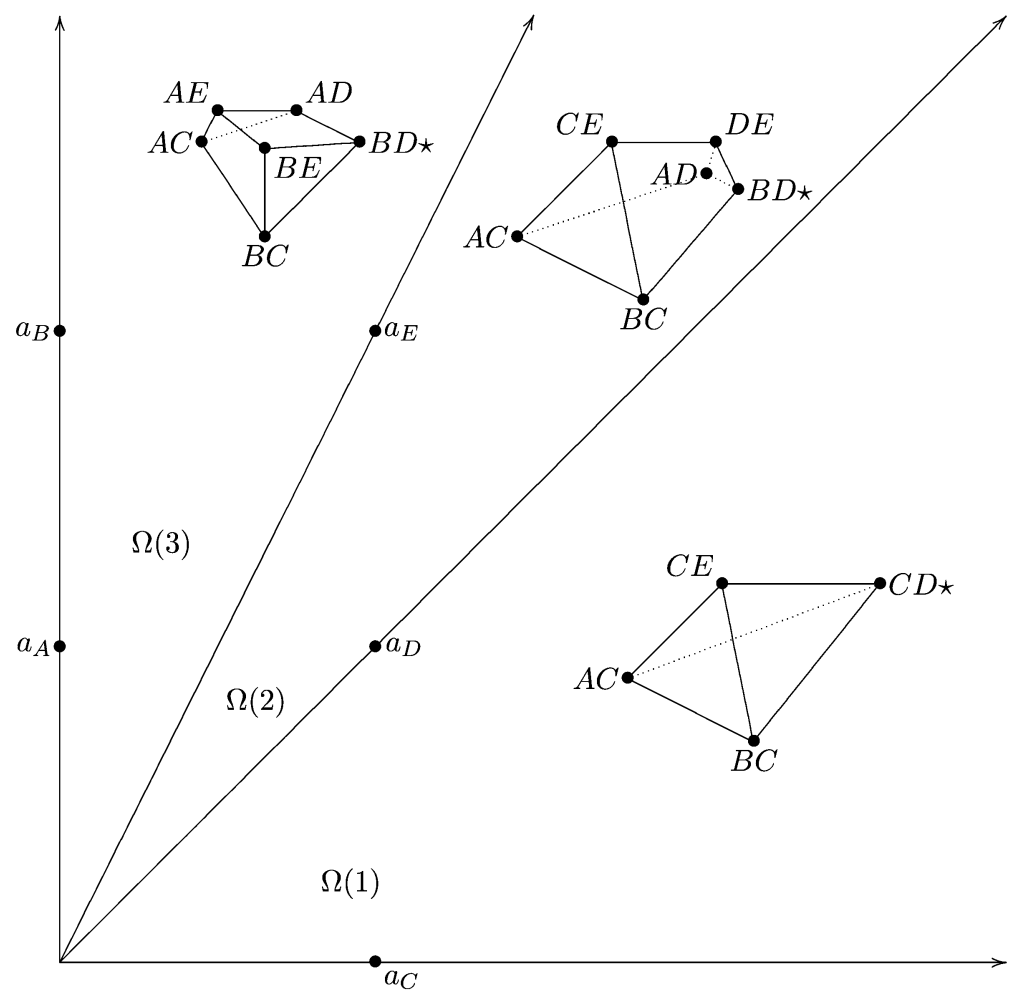

Fig. 1 The chamber decomposition of the cone $\mathcal{Q}$ for the network in Example 1.1. The cone is spanned by the columns of the matrix $\mathbf{A}$ in (5). Each of the three maximal chambers $\Omega(1), \Omega(2)$, and $\Omega$ (3) contains a picture of the corresponding 3-dimensional polyhedron $\mathcal{P}_{c}(0)$. The vertices of each polyhedron are labeled by their supports. The star “ $\star$ " indicates the unique vertex steady state, which arises from the siphon $\{A, B, E\}$ or $\{A, C, E\}$.

The two rows of $\mathbf{A}$ form a basis of $L_{\text {cons }}$. The cone $\mathcal{Q}$ is spanned by the columns of $\mathbf{A}$. The chamber decomposition of $\mathcal{Q}$ is depicted in Fig. 1. We see that the two facets of $\mathcal{Q}$ define the following ideal of $\mathbb{Q}[A, B, C, D, E]$ :

$$
\mathfrak{B}=\langle C, D, E\rangle \cap\langle A, B, D, E\rangle=\langle A C, B C, D, E\rangle .
$$

The relevant siphons are derived from $\mathfrak{M}_{G}=\left\langle A^{2} C, A D, E, B C\right\rangle$ as follows:

$$
\operatorname{Sat}\left(\mathfrak{M}_{G}, \mathfrak{B}\right)=\langle A, B C, E\rangle=\langle A, B, E\rangle \cap\langle A, C, E\rangle .
$$

Thus two of three minimal siphons in Example 1.1 are relevant. The third siphon is not relevant as its ideal $\langle C, D, E\rangle$ contains $\mathfrak{B}$. This corresponds to the fact, seen in Fig. 1, that the vectors $a_{A}$ and $a_{B}$ span a facet of $\mathcal{Q}$.

The chamber decomposition of $\mathcal{Q}$ consists of three open chambers $\Omega(1), \Omega(2)$, and $\Omega$ (3), and two rays $\Omega(12)$, and $\Omega$ (23) between the three chambers. These five chambers are encoded in the following ideals, whose generators can be read off from the vertex 
labels of the polyhedra $\mathcal{P}_{\Omega}$ in Fig. 1:

$$
\begin{aligned}
\mathfrak{B}_{\Omega(1)} & =\langle C D, C E, A C, B C\rangle, \\
\mathfrak{B}_{\Omega(12)} & =\langle D, C E, A C, B C\rangle, \\
\mathfrak{B}_{\Omega(2)} & =\langle A D, B D, D E, C E, A C, B C\rangle, \\
\mathfrak{B}_{\Omega(23)} & =\langle A D, B D, E, A C, B C\rangle, \\
\mathfrak{B}_{\Omega(3)} & =\langle A D, B D, A E, B E, A C, B C\rangle .
\end{aligned}
$$

For each chamber $\Omega$, the ideal $\operatorname{Sat}\left(\mathfrak{M}_{G}, \mathfrak{B}_{\Omega}\right)$ reveals the $\Omega$-relevant siphons. We find that $\langle A, B, E\rangle$ is $\Omega(1)$ - and $\Omega(12)$-relevant, and that $\langle A, C, E\rangle$ is $\Omega(12)-, \Omega(2)$-, $\Omega(23)$-, and $\Omega(3)$-relevant. These two siphons define a unique vertex steady state on each invariant polyhedron $\mathcal{P}_{c^{(0)}}$. Note that the vertices $F_{\{A, B, E\}}$ and $F_{\{A, C, E\}}$ coincide for polyhedra along the ray $\Omega(12)$.

The need for efficient algorithms for computing minimal siphons has been emphasized by Angeli et al. (2007), who argued that such an algorithm would allow quick verification of the hypotheses of Theorem 3.5. Cordone et al. introduced one algorithm for computing minimal siphons in Cordone et al. (2005). We advocate Theorem 3.1 as a new method for computing all minimal siphons, and Algorithm 3.6 as a direct method for identifying relevant siphons. Rather than implementing any such algorithm from scratch, it is convenient to harness existing tools for monomial and binomial primary decomposition (Dickenstein et al., 2010; Eisenbud and Sturmfels, 1996). We recommend the widely-used computer algebra system Macaulay 2 (Grayson and Stillman), and the implementations developed by Kahle (2009) and Roune (2009).

In what follows we show some snippets of Macaulay 2 code, and we discuss how they are used to compute (relevant) minimal siphons of small networks. Thereafter, we examine two larger examples, which illustrate the efficiency and speed of monomial and binomial primary decomposition. These examples support our view that the algebraic methods of Section 3 are competitive for networks whose size is relevant for research in systems biology.

Example 4.2. The following Macaulay 2 input uses the command decompose to output the minimal primes for the three examples in the Introduction.

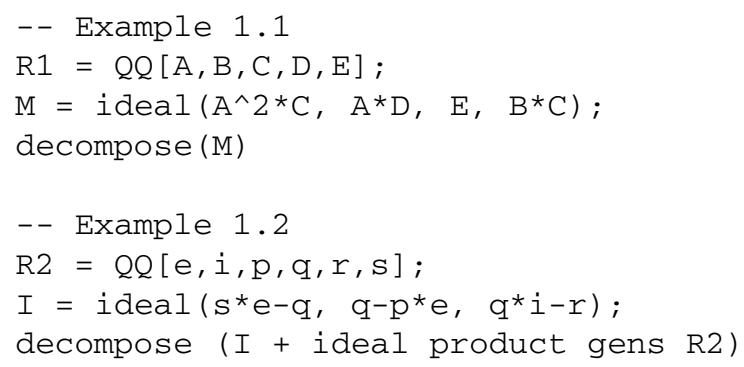


-- Example 1.3

$\mathrm{R} 3=\mathrm{QQ}\left[\mathrm{E}, \mathrm{F}, \mathrm{P}, \mathrm{S} \_0, \mathrm{X}, \mathrm{Y}\right]$;

$\mathrm{J}=$ ideal $\left(\mathrm{E} * \mathrm{~S} \_0-\mathrm{X}, \mathrm{X} *(\mathrm{E} * \mathrm{P}-\mathrm{X}), \mathrm{F}{ }^{*} \mathrm{P}-\mathrm{Y}, \mathrm{Y} *\left(\mathrm{~F}{ }^{*} \mathrm{~S} \_0-\mathrm{Y}\right)\right)$;

decompose ( $\mathrm{J}+$ ideal product gens R3)

By Theorem 3.1, the minimal siphons can be read off from the primes.

Example 4.3. We return to the chemical reaction network of Examples 1.1 and 4.1. The following Macaulay 2 code utilizes item 2 in Algorithm 3.6.

-- Example 1.1: c0-relevant siphons

$\mathrm{C} 0=\{0,0,1,1,0\}$;

$\mathrm{R}=\mathrm{QQ}[\mathrm{A}, \mathrm{B}, \mathrm{C}, \mathrm{D}, \mathrm{E}$, Weights $=>\mathrm{C} 0]$;

$I G=$ ideal $\left(A^{\wedge} 2{ }^{*} C-A * D, A * D-E, E-B * C, A * B * C * D * E\right)$;

ICons $=$ ideal $\left(C * D * E-1, A * B^{\wedge} 2 * D^{*} E^{\wedge} 2-1\right)$;

$\mathrm{BCO}=$ dual radical monomialideal leadTerm ICons;

decompose saturate (IG, BCO)

In the first line, the vector $c^{(0)}$ was chosen to represent a point in the chamber $\Omega(1)$, so the output is the unique $\Omega(1)$-relevant minimal siphon.

The next example is of a large strongly-connected chemical reaction, and the computation shows the power of monomial primary decomposition.

Example 4.4. Consider the following strongly connected network which is comprised of $s$ species, $s-1$ complexes, and $s-2$ reversible reactions:

$$
c_{1} c_{2} \leftrightarrows c_{2} c_{3} \leftrightarrows c_{3} c_{4} \leftrightarrows \cdots \leftrightarrows c_{s-1} c_{s}
$$

The number of minimal siphons satisfies the recursion $N(s)=N(s-2)+N(s-3)$, where $N(2)=2, N(3)=2$, and $N(4)=3$. For $s=50$ species, we obtain $N(50)=1,221,537$. The following Macaulay 2 code verifies this:

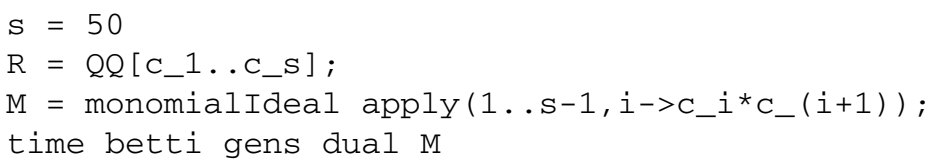

We now explain the commands that are used above. First, $\mathbb{M}$ is the monomial ideal $\mathfrak{M}_{G}$ generated by complexes, and dual outputs its Alexander dual (Miller and Sturmfels, 2005), which is the monomial ideal whose generators are the products of the speciesvariables in any minimal siphon. Secondly, betti applied to gens dual Moutputs the degrees of all the generators of dual M; these degrees are exactly the sizes of all minimal siphons. The command time allows us to see that the computation of the minimal siphons takes only a few seconds. Displayed below is a portion the output of the last command above; the list tells the number of minimal siphons of each possible size.

$$
05=\operatorname{total}: \begin{array}{rr}
0 & 1 \\
0: & 1
\end{array}
$$




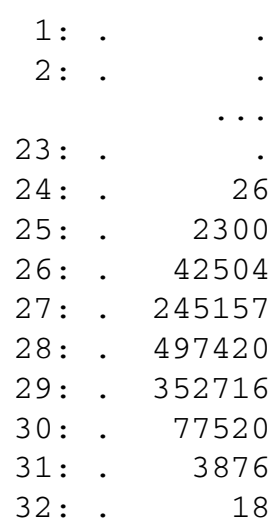

The current version of dual in Macaulay 2 uses Roune's implementation of his slice algorithm (Roune, 2009). For background on the relation of Alexander duality and primary decomposition of monomial ideals, see the textbook (Miller and Sturmfels, 2005).

Our final example aims to illustrate the computation of minimal siphons for a larger network with multiple strongly connected components.

Example 4.5. We here consider a chemical reaction network $G$ with $s=25$ species, 16 bidirectional reactions and 32 complexes. The binomials representing the 16 reactions are the adjacent $2 \times 2$-minors of a $5 \times 5$-matrix $\left(c_{i j}\right)$, and $\mathfrak{J}_{G}$ is the ideal generated by these 16 minors $c_{i, j} c_{i+1, j+1}-c_{i, j+1} c_{i+1, j}$. For this network, $L_{\text {stoi }}$ is the 16-dimensional space consisting of all matrices whose row sums and column sums are zero, and $\mathcal{Q}$ is a 9-dimensional convex polyhedral cone, namely the cone over the product of simplices $\Delta_{4} \times \Delta_{4}$.

What follows is an extension of the results for adjacent minors of a $4 \times 4$-matrix in Diaconis et al. (1998, Section 4). The ideal $\mathfrak{J}_{G}$ is not radical. Using Kahle's software (Kahle, 2009), we found that it has 103 minimal primes, of which precisely 26 contribute minimal siphons that are relevant. Up to symmetry, these 26 siphons fall into four symmetry classes, with representatives given by the following:

$$
\begin{aligned}
& Z_{1}=\left\{c_{14}, c_{21}, c_{22}, c_{23}, c_{24}, c_{32}, c_{34}, c_{42}, c_{43}, c_{44}, c_{45}, c_{52}\right\}, \\
& Z_{2}=\left\{c_{14}, c_{21}, c_{22}, c_{23}, c_{24}, c_{33}, c_{34}, c_{35}, c_{41}, c_{42}, c_{43}, c_{53}\right\}, \\
& Z_{3}=\left\{c_{14}, c_{24}, c_{31}, c_{32}, c_{33}, c_{34}, c_{42}, c_{43}, c_{44}, c_{45}, c_{52}\right\}, \\
& Z_{4}=\left\{c_{14}, c_{24}, c_{31}, c_{32}, c_{33}, c_{34}, c_{43}, c_{44}, c_{45}, c_{53}\right\} .
\end{aligned}
$$

Under the group $D_{8}$ of reflections and rotations of the matrix $\left(c_{i j}\right)$, the orbit of $Z_{1}$ consists of two siphons, and the orbits of $Z_{2}, Z_{3}$, and $Z_{4}$ each are comprised of eight siphons. The corresponding four types of minimal primes have codimensions $13,12,12$, and 12 , and degrees $1,2,3$, and 6 .

By randomly generating chambers, we found that, for every integer $r$ between 0 and 26, other than 23 and 25 , there is a point $c^{(0)}$ in $\mathcal{Q}$ such that the number of $c^{(0)}$-relevant siphons is precisely $r$. We briefly discuss this for three initial conditions. First, let $c^{(0)}$ be 
the all-ones matrix. Then $\mathcal{P}_{c}^{(0)}$ is the Birkhoff polytope which consists of all non-negative $5 \times 5$-matrices with row and column sums equal to five. In this case, all 26 minimal siphons are $c^{(0)}$-relevant: $Z_{1}$ defines a vertex, $Z_{2}$ and $Z_{3}$ define edges, and $Z_{4}$ defines a three-dimensional face of $\mathcal{P}_{c^{(0)}}$. Next, consider the following initial conditions:

$$
d^{(0)}=\left(\begin{array}{ccccc}
1 & 1 & 1 & 1 & 1 \\
1 & 1 & 1 & 1 & 1 \\
1 & 1 & 1-\epsilon & 1 & 1 \\
1 & 1 & 1 & 1 & 1 \\
1 & 1 & 1 & 1 & 1
\end{array}\right) \quad \text { and } \quad e^{(0)}=\left(\begin{array}{ccccc}
1 & 1 & 1 & 1 & 1 \\
1 & 1 & 1 & 1 & 1 \\
1 & 1 & 1+\epsilon & 1 & 1 \\
1 & 1 & 1 & 1 & 1 \\
1 & 1 & 1 & 1 & 1
\end{array}\right)
$$

where $\epsilon>0$. Again, all 26 minimal siphons are $d^{(0)}$-relevant, and $F_{Z_{1}}$ is a vertex, $F_{Z_{2}}$ and $F_{Z_{3}}$ are edges of $\mathcal{P}_{d^{(0)}}$, but now $F_{Z_{4}}$ is a five-dimensional face. Finally, for initial condition $e^{(0)}$, only two minimal siphons are $e^{(0)}$-relevant, both in the class of $Z_{1}$, and they define vertices. Thus, using the results of Anderson and Shiu (2010), we can conclude that the system (1) is persistent for $e^{(0)}$.

\section{Conclusion}

In the present paper, we gave a method that computes siphons and determines which of them are relevant. To our knowledge, this is the first automatic procedure for checking the relevance of a siphon. As noted by Angeli et al. (2007), such a procedure is desirable for verifying whether large biochemical reaction systems are persistent. Recall that persistence is the property that no species concentration tends to zero. In practice, this corresponds to the observed behavior that a substrate that is present at the beginning of an experiment will also be present in some amount for all time. There is a class of systems for which the nonrelevance of all siphons is a sufficient condition for such a system to be persistent, so our procedure can be used to verify quickly that a large network is persistent. Such a class consists of toric dynamical systems (Craciun et al., 2009). Mathematically, the claim that toric dynamical systems are persistent is the content of the Global Attractor Conjecture, and we speculate that an algebraic approach to understanding siphons may be a step toward the conjecture.

\section{Acknowledgements}

Anne Shiu was supported by a Lucent Technologies Bell Labs Graduate Research Fellowship. Bernd Sturmfels was partially supported by the National Science Foundation (DMS-0456960 and DMS-0757236). The authors acknowledge the helpful comments of anonymous reviewers, which greatly improved the paper.

Open Access This article is distributed under the terms of the Creative Commons Attribution Noncommercial License which permits any noncommercial use, distribution, and reproduction in any medium, provided the original author(s) and source are credited. 


\section{References}

Adleman, L., Gopalkrishnan, M., Huang, M.-D., Moisset, P., Reishus, D., 2008. On the mathematics of the law of mass action. arXiv:0810.1108.

Anderson, D., 2008. Global asymptotic stability for a class of nonlinear chemical equations. SIAM J. Appl. Math. 68, 1464-1476.

Anderson, D., Shiu, A., 2010. Persistence of deterministic population processes and the global attractor conjecture. SIAM J. Appl. Math. (to appear). arXiv:0903.0901.

Angeli, D., Sontag, E., 2006. Translation-invariant monotone systems, and a global convergence result for enzymatic futile cycles. Nonlinear Anal. Ser. B: Real World Appl. 9, 128-140.

Angeli, D., De Leenheer, P., Sontag, E., 2007. A Petri net approach to persistence analysis in chemical reaction networks. In: Queinnec, I., Tarbouriech, S., Garcia, G., Niculescu, S.-I. (Eds.), Biology and Control Theory: Current Challenges, Lecture Notes in Control and Information Sciences, vol. 357, pp. 181-216. Springer, Berlin.

Chavez, M., 2003. Observer design for a class of nonlinear systems, with applications to chemical and biological networks. Ph.D. Thesis, Rutgers University.

Cordone, R., Ferrarini, L., Piroddi, L., 2005. Enumeration algorithms for minimal siphons in Petri nets based on place constraints. IEEE Trans. Syst. Man Cybern. Part A 35(6), 844-854.

Cox, D., Little, J., O'Shea, D., 2007. Ideals, Varieties and Algorithms, 3rd edn., Undergraduate Texts in Mathematics. Springer, New York.

Craciun, G., Dickenstein, A., Shiu, A., Sturmfels, B., 2009. Toric dynamical systems. J. Symb. Comput. 44(11), 1551-1565.

Craciun, G., Pantea, C., Rempala, G., 2009. Algebraic methods for inferring biochemical networks: a maximum likelihood approach. Comput. Biol. Chem. 33(5), 361-367.

Diaconis, P., Eisenbud, D., Sturmfels, B., 1998. Lattice walks and primary decomposition. In: Sagan, B.E., Stanley, R.P. (Eds.), Mathematical Essays in Honor of Gian-Carlo Rota, pp. 173-193. Birkhäuser, Basel.

Dickenstein, A., Matusevich, L., Miller, E., 2010. Combinatorics of binomial primary decomposition. Math. Z. (to appear). arXiv:0803.3846.

Eisenbud, D., Sturmfels, B., 1996. Binomial ideals. Duke Math. J. 84, 1-45.

Gawrilow, E., Joswig, M., 2000. Polymake: a framework for analyzing convex polytopes. In: PolytopesCombinatorics and Computation, Oberwolfach, 1997, DMV Sem., vol. 29, pp. 43-73. Birkhäuser, Basel.

Grayson, D., Stillman, M. Macaulay 2, a software system for research in algebraic geometry, www.math.uiuc.edu/Macaulay2/.

Kahle, T., 2009. Decompositions of binomial ideals. arXiv:0906.4873.

Manrai, A., Gunawardena, J., 2008. The geometry of multisite phosphorylation. Biophys. J. 95, 55335543.

Miller, E., Sturmfels, B., 2005. Combinatorial Commutative Algebra, Graduate Texts in Mathematics. Springer, New York.

Roune, B., 2009. The slice algorithm for irreducible decomposition of monomial ideals. J. Symb. Comput. 44(4), 358-381.

Siegel, D., MacLean, D., 2004. Global stability of complex balanced mechanisms. J. Math. Biol. 27, 89110. 